Pacific Journal of Mathematic 


\title{
GENERALIZED SYLOW TOWER GROUPS
}

\author{
J. B. DERR
}

\begin{abstract}
A well-known theorem of $P$. Hall states that a finite group $G$ is solvable if, and only if $G$ possesses a complete set of permutable Sylow subgroups. Our goal here is to investigate finite (solvable) groups whose Sylow subgroups are related by a normalizer condition $(N)$. The presence of property $(N)$ for a group $G$ implies that $G$ has structure similar to a Sylow tower group.
\end{abstract}

Some well-known classes of finite groups can be described in terms of Sylow structure. For example, a finite nilpotent group is characterized by the property that distinct Sylow subgroups centralize one another. And P. Hall [3] has shown that a finite solvable group is characterized by the existence of a complete set of pairwise permutable Sylow subgroups. More recently, Huppert [6] has investigated groups with property $(V)$ : any pair of Sylow subgroups having coprime orders permute as subgroups.

This work investigates groups in connection with the following normalizer condition $(N)$. If $\mathscr{S}$ is a collection of subgroups of a finite group, we say $\mathscr{S}$ satisfies $(N)$ provided: for any pair of subgroups in $\mathscr{S}$ having coprime orders, at least one of the subgroups normalizes the other. The known structures of solvable and nilpotent groups motivate our choices for the set $\mathscr{S}$. The main results are listed as $1.7-1.8,2.5$ and 3.1 .

All groups considered here are finite and the following notation is used: If $G$ is a group then

$\pi(G)$ is the number of prime divisors of the order of $G$,

$l(G)$ is the nilpotent (Fitting) length of $G$,

$Z(G)$ is the center of $G$,

$\phi(G)$ is the Frattini subgroup of $G$.

If $H$ is a subgroup of $G$ then

$N_{G}(H)$ is the normalizer of $H$ in $G$,

$C_{G}(H)$ is the centralizer of $H$ in $G$.

If $p$ is a prime, $G_{p}$ denotes a Sylow $p$-subgroup of $G$.

1. Generalized Sylow tower groups. Let $G$ be a group with order $p_{1}^{\alpha_{1}} \cdots p_{r}^{\alpha_{r}}$, where $p_{1}, \cdots, p_{r}$ are distinct primes and $\alpha_{1}, \cdots, \alpha_{r}$ are positive integers. For $i=1, \cdots, r$ let $G_{i}$ denote a Sylow $p_{i}$-subgroup of $G$. The collection of subgroups $\mathscr{S}=\left\{G_{1}, \cdots, G_{r}\right\}$ is called a complete set of Sylow subgroups of $G$. If the subgroups of $\mathscr{S}$ are pairwise permutable as subgroups (that is, if $G_{i} G_{j}=G_{j} G_{i}, 1 \leqq i, j \leqq r$ ), 
$\mathscr{S}$ is called a Sylow basis of $G$. The collection of $2^{r}$ subgroups of $G$ formed by all products of subgroups in a Sylow basis of $G$ is a Sylow system of $G$.

If a complete set $\mathscr{S}$ of Sylow subgroups of $G$ satisfies $(N)$, we call $G$ a generalized Sylow tower group (GSTG). In this case, $\mathscr{S}$ is a Sylow basis of $G$ and hence $G$ is solvable. On the other hand, one can easily show that every Sylow tower group is necessarily a GSTG.

Lemma 1.1. If $G$ is a GSTG, then every Sylow basis of $G$ satisfies $(N)$.

Proof. Let $\mathscr{S}=\left\{G_{1}, \cdots, G_{r}\right\}$ be a Sylow basis of $G$ satisfying $(N)$ and $\mathscr{T}$ any Sylow basis of $G$. By [4, p. 320], there exists an element $g \in G$ with $\mathscr{T}=\left\{G_{1}^{g}, \cdots, G_{r}^{o}\right\}$. Then $\mathscr{T}$ clearly satisfies $(N)$ since $\mathscr{S}$ does.

Let $G$ be a generalized Sylow tower group and $\mathscr{S}$ a Sylow basis of $G$ which satisfies $(N)$. If $R$ is a relation on the set of all primes and if the Sylow $p$-subgroup of $G$ in $\mathscr{S}$ normalizes the Sylow $q$-subgroup of $G$ in $\mathscr{S}$ whenever $p R q$, then we call $G$ a generalized Sylow tower group of complexion $R$. The preceding lemma shows that the complexion of $G$ is independent of the Sylow basis chosen. It should be noted that a given GSTG may have many complexions.

In the sequel, $R$ denotes a relation on the set of all primes.

Proposition 1.2. Let $G$ be a GSTG of complexion $R$. If $K$ is a subgroup of $G$, then $K$ is also a GSTG of complexion $R$.

Proof. Let $\mathscr{S}=\left\{G_{1}, \cdots, G_{r}\right\}$ be a Sylow basis of $G$ which satisfies $(N)$. If $K$ is a Hall subgroup of $G$, then $K$ is conjugate to the subgroup of $G$ formed by the product of those subgroups in $\mathscr{S}$ whose orders divide $|K|$. Suppose then $K=\left(G_{i_{1}} \cdots G_{i_{n}}\right)^{g}$, some $g \in G$. Clearly $\left\{G_{i_{1}}^{g}, \cdots, G_{i_{n}}^{g}\right\}$ is a Sylow basis of $K$ satisfying $(N)$ and $K$ has complexion $R$.

If $\pi(K)<\pi(G)$, the assertion follows by induction. Suppose now that $\pi(K)=\pi(G)$ and let $\mathscr{K}=\left\{K_{1}, \cdots, K_{r}\right\}$ be any Sylow basis of $K$. By a result of P. Hall [4, p. 321], there is an element $x \in G$ such that $K_{i}=K \cap G_{i}^{x}$ for $i=1, \cdots, r$. It is now clear that $\mathscr{K}$ satisfies $(N)$ and $K$ has complexion $R$.

Proposition 1.3. A homomorphic image of a GSTG with complexion $R$ is again a GSTG of complexion $R$.

Proof. Let $\left\{G_{1}, \cdots, G_{r}\right\}$ be any Sylow basis of $G$. Let $\tau$ be a homomorphism of $G$ onto $H$ and denote the image of $G_{i}$ under $\tau$ by 
$H_{i}$ (for $i=1, \cdots, r$ ). Then the collection of the nontrivial $H_{i}$ is a Sylow basis of $H$ which satisfies $(N)$. Clearly $H$ has the same normalizing structure as $G$.

Let $A$ be the symmetric group of degree 4 and $B$ the normal subgroup of $A$ having order 4 . Then both $B$ and $A / B$ are GSTG's of the same complexion. But $A$ is clearly not a GSTG since $\pi(A)=2$ and $A$ has no normal Sylow subgroup. In this respect, a GSTG has structure similar to a nilpotent group.

Proposition 1.4. Let $Z_{\infty}$ denote the hypercenter of a group $G$. If $G / Z_{\infty}$ is a GSTG, then $G$ is a GSTG.

Proof. Let $Z$ denote the center of $G$ and show that $G$ is a GSTG whenever the factor group $G / Z$ is a GSTG. Suppose $G / Z$ is a GSTG and $\mathscr{S}=\left\{G_{1}, \cdots, G_{r}\right\}$ is a complete set of Sylow subgroups of $G$ chosen so that $\mathscr{S}^{*}=\left\{G_{1} Z / Z, \cdots, G_{r} Z \mid Z\right\}$ satisfies $(N)$. For integers $i$ and $j$ with $1 \leqq i, j \leqq r$, let $G_{i} Z / Z$ normalize $G_{j} Z / Z$. Then $G_{i}$ normalizes $G_{j}$ and it follows that $\mathscr{S}$ satisfies $(N)$.

It is easy to see that $G$ need not have the same complexion as $G / Z_{\infty}$. The importance of the complexion of a GSTG arises in connection with the direct product of GSTG's. If the direct product of groups $A$ and $B$ is a GSTG of complexion $R, 1.2$ shows that both $A$ and $B$ are GSTG's of complexion $R$. The converse is the following.

Proposition 1.5. If $H$ and $K$ are GSTG's of (the same) complexion $R$, the direct product $H \times K$ is also a GSTG of complexion $R$.

Proof. Let $\mathscr{C}$ and $\mathscr{K}$ be (resp.) Sylow bases of $H$ and of $K$. Construct a complete set of Sylow subgroups of $H \times K$, say $\mathscr{D}$, by forming the appropriate products of subgroups from $\mathscr{H}$ and $\mathscr{K}$. Then $\mathscr{D}$ satisfies $(N)$ and $H \times K$ is a GSTG of complexion $R$.

Proposition 1.6. Let $H$ and $K$ be normal subgroups of $G$. If the factor groups $G / H$ and $G / K$ are both GSTG's of complexion $R$, then $G / H \cap K$ is a GSTG of complexion $R$.

Proof. This follows immediately since $G / H \cap K$ is isomorphic to a subgroup of $G / H \times G / K$.

If $R$ is any relation on the set of all primes, then 1.3 and 1.6 together show that the class of all GSTG's with complexion $R$ is a formation in the sense of Gaschütz. However, since the direct product of GSTG's need be a GSTG, the class of all GSTG's is not a formation. 
An interesting property of GSTG's and a connection between GSTG's and Sylow tower groups may now be shown.

THEOREM 1.7. Let $G$ be a GSTG. Then the nilpotent length of $G$ is at most $\pi(G)$, the number of distinct prime divisors of the order of $G$.

Proof. If $\pi(G) \leqq 2, G$ has a normal Sylow subgroup and clearly $l(G) \leqq \pi(G)$. Suppose $\pi(G)=k \geqq 3$ and let $\mathscr{S}$ be a Sylow basis of $G$ which satisfies $(N)$. We proceed by induction on $|G|$.

Suppose first that $G$ has a nontrivial, normal Sylow subgroup $T$. By induction, the factor $G / T$ then has nilpotent length at most $k-1$. Hence $G$ has nilpotent length at most $k=\pi(G)$.

Assume now that $G$ has no nontrivial, normal Sylow subgroup. Since $G$ is solvable, $G$ has a nontrivial normal $p$-subgroup for some prime $p$. Let $P$ denote the maximal normal $p$-subgroup of $G$ and $G_{p}$ the Sylow $p$-subgroup of $G$ belonging to $\mathscr{S}$. Since $G_{p} \sharp G$, there is a prime $q, q \neq p$, and Sylow $q$-subgroup $G_{q}$ belonging to $\mathscr{S}$ for which $G_{p}$ normalizes $G_{q}$. Consequently $G_{q} \leqq G_{G}(P)$ and so $\pi\left(C_{G}(P)\right) \geqq 2$.

Let $P_{0}=C_{G}(P) \cap P$, the maximal normal $p$-subgroup of $C_{G}(P)$, and let $V / P_{0}$ denote a nontrivial normal subgroup of $C_{G}(P) / P_{0}$ of prime power order $r^{\alpha}$ ( $r$ some prime). By the maximality of $P_{0}, r \neq p$. If $W / P_{0}$ denotes the maximal normal $r$-subgroup of $C_{G}(P) / P_{0}$, then $W / P_{0}$ is characteristic in $C_{G}(P) / P_{0}$ and hence $W \leqq G$. Since $W \leqq C_{G}(P)$ and $W / P_{0}$ is an $r$-group, $W$ has normal Sylow $r$-subgroup $R$. Then $R$ is characteristic in $W$, hence normal in $G$.

By induction, the factors $G / P$ and $G / R$ both have nilp. length at most $k$. Since $P \cap R=1, G$ is isomorphic to a subgroup of the direct product $G / P \times G / R$. Therefore $l(G) \leqq \max \{l(G / P), l(G / R)\} \leqq k=\pi(G)$.

THEOREM 1.8. If $G$ is a GSTG and $l(G)=\pi(G)$, then $G$ is a Sylow tower group.

For the proof of this, the following lemma is helpful.

Lemma. Let $G$ be a Sylow tower group with $l(G)=\pi(G)$. If $S$ and $T$ are distinct Sylow subgroups of $G$, then $S$ and $T$ do not centralize one another.

Proof. Suppose the assertion false and let $G$ be a counterexample of minimal order. Then $G$ is a Sylow tower group with $l(G)=\pi(G)=$ $n \geqq 3$. Let

$$
1 \triangleleft S_{1} \triangleleft S_{2} S_{1} \triangleleft \cdots \triangleleft S_{n} S_{n-1} \cdots S_{1}=G
$$


be a Sylow tower of $G$, where $S_{i}$ denotes a Sylow $p_{i}$-subgroup of $G$ for $i=1,2, \cdots, n$. The factor group $G / S_{1}$ is a Sylow tower group with $l\left(G / S_{1}\right)=n-1=\pi\left(G / S_{1}\right)$. Therefore, by the minimality of $|G|$, no distinct Sylow subgroups of $G / S_{1}$ centralize one another.

By assumption, some Sylow $p_{j}$-subgroup $T$ of $G$ centralizes some Sylow $p_{k}$-subgroup $V$ of $G$ (for some $j, k$ distinct). Then $T S_{1} / S_{1}$ centralizes $V S_{1} / S_{1}$ and consequently one of these Sylow subgroups of $G / S_{1}$ is trivial. Suppose $T S_{1} / S_{1}$ is trivial. Then $j=1$ and it follows that $S_{k}$ centralizes $S_{1}$. Choose $m>1$ to be the smallest integer for which $p_{m}$ divides $\left|C_{G}\left(S_{1}\right)\right|$ and put $R=S_{m} \cdot S_{m-1} \cdots S_{1} \cap C_{G}\left(S_{1}\right)$. Then $R$ is normal in $G$ and has order $p_{1}^{\alpha} p_{m}^{\beta}$. If $W$ denotes the Sylow $p_{m}$-subgroup of $R$, then $W$ is characteristic in $R$ and normal in $G$.

We may assume $W$ is not a Sylow subgroup of $G$. Otherwise $W S_{1}$ is a normal nilpotent subgroup of $G$ and $l(G) \leqq n-1$, which is a contradiction. Hence $S_{k} W / W$ is a nontrivial Sylow $p_{k}$-subgroup of $G / W$ which centralizes $S_{1} W / W$. Since $\pi(G / W)=n$, the minimality of $G$ implies $l(G / W)<n$.

Since $W \cap S_{1}=1, G$ is isomorphic to a subgroup of $G / W \times G / S_{1}$. Therefore $l(G) \leqq \max \left\{l\left(G / S_{1}\right), l(G / W)\right\}<n$, which is the desired contradiction.

Proof of 1.8. Let $G$ be a GSTG with $l(G)=\pi(G)$. If $\pi(G) \leqq 2$, $G$ has a normal Sylow subgroup and clearly $G$ is a Sylow tower group. Take $l(G)=\pi(G)=n \geqq 3$ and proceed by induction.

Suppose first that $G$ has a nontrivial normal Sylow subgroup $K$. Then $G / K$ is a GSTG with $l(G / K)=n-1=\pi(G / K)$ and induction implies $G / K$ is a Sylow tower group. Then $G$ is also a Sylow tower group as asserted. Now assume $G$ has no nontrivial normal Sylow subgroup. As in the proof of 1.7, $G$ then has nontrivial normal subgroups $M_{1}$ and $M_{2}$ with $\left|M_{1}\right|=p^{\alpha},\left|M_{2}\right|=q^{\beta}$ for $p, q$ distinct primes. If both $G / M_{1}$ and $G / M_{2}$ have nilpotent length less than $n$, then $l(G)<n$, a contradiction. Therefore we may assume $l\left(G / M_{1}\right)=n$. Since $\pi\left(G / M_{1}\right)$ is also $n$, induction shows $G / M_{1}$ is a Sylow tower group. Let

$$
\overline{1}=M_{1} / M_{1} \triangleleft S_{1} M_{1} / M_{1} \triangleleft S_{2} S_{1} M_{1} / M_{1} \triangleleft \cdots \triangleleft S_{n} \cdots S_{1} M_{1} / M_{1}=G / M_{1}
$$

be a Sylow tower of $G / M_{1}$, where $S_{i}$ is a Sylow $p_{i}$-subgroup of $G$ for $i=1,2, \cdots, n$. In addition, choose $S_{n}$ to be the Sylow $p_{n}$-subgroup of $G$ belonging to $\mathscr{S}$, where $\mathscr{S}$ is a Sylow basis of $G$ which satisfies $(N)$ and contains $S_{1}$.

We first show that $M_{1} \leqq S_{n}$. If this were not the case, then $M_{1} \leqq S_{k}$ for some $k<n$. Then $H=S_{k} \cdots S_{2} S_{1}$ is a normal Hall subgroup of $G$ and, by induction, $H$ is a Sylow tower group. Therefore 
$H$ has a normal Sylow subgroup $K$. But then $K$ is a normal Sylow subgroup of $G$, which contradicts our assumption about $G$.

By our choice of $S_{1}$ and $S_{n}$, either $S_{1} \leqq N_{G}\left(S_{n}\right)$ or $S_{n} \leqq N_{G}\left(S_{1}\right)$. If $S_{n} \leqq N_{G}\left(S_{1}\right),\left[S_{1}, M_{1}\right]=1$ and consequently $S_{1} \triangleleft G-$ which is impossible. Therefore we may assume $S_{1} \leqq N_{G}\left(S_{n}\right)$. Then the Sylow $p_{1}$ subgroup $S_{1} M_{1} / M_{1}$ of $G / M_{1}$ centralizes the Sylow $p_{n}$-subgroup $S_{n} / M_{1}$ of $G / M_{1}$, which contradicts the lemma. Therefore $G$ must have a normal Sylow subgroup and the theorem is proved.

The following construction shows the existence of a GSTG $G$ with nilpotent length $n$ and $\pi(G)=m+n$, where $m$ and $n$ are arbitrary nonnegative integers and $n \neq 0$. This shows in particular that the inequality of Theorem 1.7 cannot be improved.

If $A$ and $B$ are groups, let $A$ or $B$ denote the wreath product of $A$ by $B$. For nonnegative integers $m$ and $n$ with $n \neq 0$, choose distinct primes $p_{1}, \cdots, p_{m+1}, q_{1}, \cdots, q_{n-1}$. Let $D$ be the cyclic group of order $p_{1} \cdots p_{m+1}$ and $C_{j}$ the cyclic group of order $q_{j}$ (for $j=$ $1, \cdots, n-1)$. Then the repeated wreath product

$$
G=\left[\cdots\left(\left(D \text { wr } C_{1}\right) \text { wr } C_{2}\right) \cdots\right] \text { wr } C_{n-1}
$$

is a GSTG with $l(G)=n$ and $\pi(G)=m+n$.

2. $N$-groups. In the preceding section groups for which some complete set of Sylow subgroups satisfies $(N)$ were examined. Now we examine groups with the property that every complete set of Sylow subgroups satisfies $(N)$. This is equivalent to demanding that the collection of all Sylow subgroups of a group satisfies $(N)$. We call such groups $N$-groups.

Any nilpotent group extended by a $p$-group is an $N$-group. And clearly every $N$-group is a GSTG and hence solvable. An $N$-group need not be a Sylow tower group however, as the following example shows. For $m$ and $n$ any positive integers, let $\operatorname{wr}(m, n)$ denote the wreath product of a cyclic group of order $m$ by a cyclic group of order $n$. Let $p_{1}, p_{2}$ and $p_{3}$ be distinct primes and put $A=\operatorname{wr}\left(p_{1}, p_{2}\right)$, $B=\operatorname{wr}\left(p_{3}, p_{1}\right)$ and $C=\operatorname{wr}\left(p_{2}, p_{3}\right)$. Then the direct product of $A, B$ and $C$ is an $N$-group which is not a Sylow tower group.

Since any two Sylow bases of a solvable group are conjugate [4, p. 321], the following holds.

Proposition 2.1. If $G$ is an $N$-group and some Sylow p-subgroup of $G$ normalizes some Sylow q-subgroup of $G$, then every Sylow psubgroup of $G$ normalizes every Sylow $q$-subgroup of $G$ ( $p$ and $q$ distinct primes). 
In many respects $N$-groups behave like GSTG's. Define an $N$ group $G$ to have complexion $R$ when $G$ considered as a GSTG has complexion $R$. Then 1.2-1.6 remain valid when "GSTG" is replaced by " $N$-group". Therefore the class of all $N$-groups having complexion $R$ is a formation. This formation will not necessarily be saturated, since $G$ need not be an $N$-group whenever $G / \phi(G)$ is an $N$-group (see [6, p. 265]).

Although $G$ need not be an $N$-group whenever $G / \dot{\phi}(G)$ is an $N$ group, the following holds.

Proposition 2.2. If $G$ is a group of order $p^{\alpha} q^{\beta}$, where $p$ and $q$ are primes, and $G / \phi(G)$ is an $N$-group, then $G$ is an $N$-group.

Proof. We may assume that $G / \dot{\phi}(G)$ has normal Sylow $p$-subgroup $G_{p} \dot{\phi}(G) / \phi(G)$, where $G_{p}$ is any Sylow $p$-subgroup of $G$. Let $W$ be the normalizer of $G_{p}$ in $G$. If $W=G, G_{p}$ is normal in $G$ and $G$ is an $N$ group. Otherwise $W$ lies in some maximal subgroup $M$ of $G$. Let $x$ be an element of $G$ not in $M$. Since

$$
G_{p} \dot{\phi}(G) / \phi(G) \triangleleft G / \phi(G), \quad G_{p}^{x} \leqq G_{p}^{x} \dot{\phi}(G) \leqq M
$$

and there is an element $y \in M$ with $G_{p}^{y}=G_{p}^{x}$. Then $x \in M$, which is impossible.

A similar argument establishes the following.

Proposition 2.3. If $G / \phi(G)$ is an $N$-group and all maximal subgroups of $G$ are $N$-groups, then $G$ is an $N$-group.

Unlike GSTG's, every $N$-group necessarily satisfies property $(V)$ of Huppert. This observation leads to an upper bound for the nilpotent length of an $N$-group.

Proposition 2.4. If $G$ is an $N$-group, then $l(G) \leqq 2$.

Proof. Since $G$ satisfies property ( $V)$, Satz 2 [6, p. 253] shows that $G / \phi(G)$ is isomorphic to a subgroup, $H$ (say), of a direct product of groups $T_{1}, \cdots, T_{n}$, where $\left|T_{i}\right|=p_{\imath}^{a} q_{i}^{b} i$ with $p_{i}$ and $q_{i}$ primes. For each integer $k, 1 \leqq k \leqq n$, define the homomorphism $\pi_{k}$ of $H$ into $T_{k}$ by $\pi_{k}\left(t_{1} \cdots t_{n}\right)=t_{k} ; t_{1} \cdots t_{n} \in H$ and $t_{i} \in T_{i}$ for each $i$. Now $\pi_{k}(H)$ is an $N$-group, being a homomorphic image of $G$. And since $\pi_{k}(H)$ is a subgroup of $T_{k}$, the nilpotent length of $\pi_{k}(H)$ is at most 2 . Therefore $l(G)=l(H) \leqq \max _{1 \leqq k \leqq n}\left\{l\left(\pi_{k}(H)\right)\right\} \leqq 2$.

$N$-groups may now be described relative to property $(V)$. 
THEOREM 2.5. Let $G$ satisfy $(V)$. Then $G$ is an $N$-group if, and only if

( i ) $G$ is a partially complemented extension of a nilpotent group $H$ by a nilpotent group $K$, and

(ii) for distinct primes $p$ and $q$, the Sylow p-subgroup $H_{p}$ of $H$ normalizes the Sylow q-subgroup $K_{q}$ of $K$ or the Sylow $q$-subgroup $H_{q}$ of $H$ normalizes the Sylow p-subgroup $K_{p}$ of $K$.

Proof. Let $G$ be an $N$-group with nilpotent length 2. Then [5, p. 211] there exist nilpotent subgroups $H$ and $K$ of $G$ with $G=H K$, $H \triangleleft G$. To verify (ii), let $p$ and $q$ be any distinct prime divisors of ( $H|| G / H \mid,) . \quad L_{1}=H_{p} K_{p} K_{q}$ is an $N$-group of order $p^{\alpha} q^{\beta}$ and hence $K_{q} \leqq N_{G}\left(H_{p} K_{p}\right)$ or $H_{p} K_{p} \leqq N_{G}\left(K_{q}\right)$. We may assume $K_{q} \leqq N_{G}\left(H_{p} K_{p}\right)$ and $H_{p} K_{p} \geqq N_{G}\left(K_{q}\right)$. Since $L_{1}$ and $G$ have the same complexion, this means $H_{q} K_{q}$ normalizes $H_{p} K_{p}$. Similarly, considering $L_{2}=H_{q} K_{q} K_{p}$ we may assume $H_{p} K_{p}$ normalizes $H_{q} K_{q}$. Therefore $H_{p} K_{p}$ and $H_{q} K_{q}$ centralize one another and we have established (ii).

For the converse, let $G$ satisfy $(V)$ and both (i) and (ii). If $G$ is a $p^{\alpha} q^{3}$ group, it easily follows that $G$ must be an $N$-group.

Suppose now that $\pi(G)=k \geqq 3$ and let $p$ and $q$ be prime factors of the order of $G$. Let $G_{p}$ and $G_{q}$ be any Sylow $p$ - and $q$-subgroups of $G$ (respectively). Then by Sylow arguments

$$
\begin{array}{lll}
G_{p}=H_{p} K_{p}^{x} & \text { for some } & x \in G \\
G_{q}=H_{q} K_{q}^{y} & \text { for some } & y \in G .
\end{array}
$$

Since $G$ satisfies $(V), L=\left(H_{p} K_{p}\right)\left(H_{q} K_{q}^{y x^{-1}}\right)$ is a subgroup of $G$. $L$ satisfies both (i) and (ii) and hence, by induction, is an $N$-group. Therefore $H_{p} K_{p} \leqq N_{G}\left(H_{q} K_{q}^{y x^{-1}}\right)$ or $H_{q} K_{q}^{y x^{-1}} \leqq N_{G}\left(H_{p} K_{p}\right)$. Then $G_{p}$ normalizes $G_{q}$ or $G_{q}$ normalizes $G_{p}$ and we have shown $G$ is an $N$-group.

In the previous theorem, if all Sylow subgroups of $G$ are abelian, then "partial complement" can be replaced by "complement". An example can be given to show that "partial complement" cannot be improved to "complement" in general.

3. Strongly Sylow towered groups. Since every finite solvable group possesses Sylow systems, it seems worth-while to consider property $(N)$ in connection with these collections of subgroups. If $G$ is a solvable group and some Sylow system of $G$ satisfies $(N)$, we call $G$ a strongly Sylow towered group (SSTG). It is easily shown that every SSTG is necessarily a Sylow tower group. However a SSTG need not be an $N$-group. For example, the holomorph of the cyclic group of order 7 is an SSTG but not an $N$-group. 
The inheritence properties of SSTG's resemble those of GSTG's. For instance, subgroups and homomorphic images of SSTG's are again SSTG's and the direct product of SSTG's with similar normalizing structures is again an SSTG.

Theorem 3.1. A group $G$ is an SSTG if, and only if, $G$ is a split extension of a nilpotent group $A$ by a nilpotent group $B$ where $A$ and $B$ have coprime orders and either $A$ or $B$ is a p-group.

Proof. Let $\mathscr{S}$ be a Sylow system of $G$ which satisfies $(N)$. Let $S$ and $T$ denote any distinct nonnormal Sylow subgroups of $G$ belonging to $\mathscr{S}$ and let $S^{\prime}$ and $T^{\prime}$ be (respectively) the complements of $S$ and $T$ in $\mathscr{S}$. Since $\mathscr{S}$ satisfies $(N)$, both $S^{\prime}$ and $T^{\prime}$ are normal in $G$. Furthermore, we may assume $S$ normalizes $T$. Then $[S, T] \leqq T^{\prime} \cap T=1$ and hence any distinct nonnormal Sylow subgroups of $G$ in $\mathscr{S}$ centralize one another. Let $A$ denote the product of all normal Sylow subgroups of $G$ and $B$ the product of the nonnormal Sylow subgroups of $G$ in $\mathscr{S}$. Then $A$ and $B$ are nilpotent and $G=A \cap B=1$ and $A \triangleleft G$.

If $A$ or $B$ is a $p$-group, we are done. Assume not and let $\pi(A)=$ $k \geqq 2, \pi(B)=l \geqq 2$. Let $N_{1}, \cdots, N_{k}$ be the normal Sylow subgroups of $G$ and $S_{1}, \cdots, S_{l}$ the nonnormal Sylow subgroups of $G$ in $\mathscr{S}$. Since $S_{1} \ggg G$, we may assume $N_{1} \nsubseteq N_{G}\left(S_{1}\right)$. Then, for any positive integers: $i, j \geqq 2, N_{1} S_{j} \leqq N_{G}\left(N_{i} S_{1}\right)$ or $N_{i} S_{1} \leqq N_{G}\left(N_{1} S_{j}\right)$. Consequently we have $N_{i} \leqq C_{G}\left(S_{j}\right)$ whenever $i, j \geqq 2$. It follows that $N_{1} \geqq N_{G}\left(S_{j}\right) ; j=$ $1, \cdots, l$.

Now suppose $N_{i}$ does not normalize $S_{1}$, some $i \geqq 2$. Since $N_{1} S_{1}$ and $N_{i} S_{2}$ are subgroups of $G$ belonging to $f$, either $N_{1} S_{1} \leqq N_{G}\left(N_{i} S_{2}\right)$ or $N_{i} S_{2} \leqq N_{G}\left(N_{1} S_{1}\right)$. Then $N_{1} \leqq C_{G}\left(S_{2}\right)$ or $N_{i} \leqq C_{G}\left(S_{1}\right)$, a contradiction. Therefore we may assume $N_{i}$ normalizes $S_{1}$ for all $i \geqq 2$. Then $H=N_{2} \cdots N_{k} S_{1} \cdots S_{l}$ is a nilpotent subgroup of $G$ and $G$ is the split extension of $N_{1}$ by $H$.

The converse follows easily. For, if $G$ is the split extension of a $p$-group $A$ by a nilpotent group $B$ of coprime order and having Sylow subgroups $B_{1}, \cdots, B_{n}$, then the Sylow basis $\left\{A, B_{1}, \cdots, B_{n}\right\}$ generates a Sylow system of $G$ which satisfies $(N)$. Next, suppose $G$ is the split extension of a nilpotent group $A$ by a $p$-group $B$ with $(|A|, p)=1$. If $A_{1}, \cdots, A_{n}$ are the Sylow subgroups of $A$, then $\left\{A_{1}, \cdots, A_{n}, B\right\}$ generates a Sylow system of $G$ which satisfies $(N)$.

The diagram below illustrates the connections between the classes of solvable groups considered here and other well-known classes. 


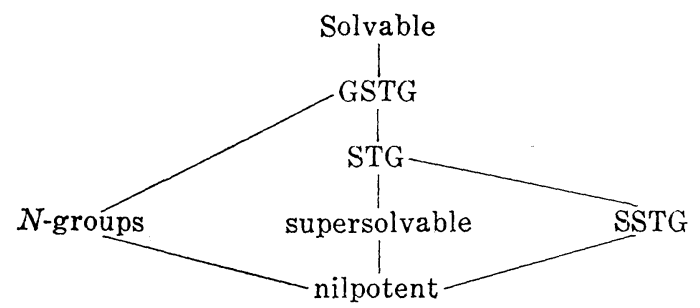

The contents of this paper formed part of the author's $\mathrm{Ph}$. D. thesis at Michigan State University in East Lansing, Michigan. It is a pleasure to have this opportunity to thank my thesis director, W.E. Deskins, for his help in the preparation of this thesis.

\section{BIBLIOGRAPHY}

1. R. Baer, Sylowturmgruppen, Math. Zeit 69 (1958), 239-246.

2. P. Hall, A note on soluble groups, J. London Math. Soc. 3 (1928), 98-105.

3. - A characteristic property of soluble groups, J. London Math. Soc. 12 (1937), 198-200.

4. On the Sylow systems of a soluble group, Proc. London Math. Soc. 43 (1937), 316-323.

5. - The construction of soluble groups, J. Reine Angew. Math. 182 (1940), 206-214.

6. B. Huppert, Zur Sylowstruktur auflösbarer Gruppen II, Arkiv der Math. 15 (1964), 251-257.

7. W. R. Scott, Group theory, Prentice Hall, Englewood Cliffs, New Jersey, 1964.

Received May 8, 1969.

The University of New Mexico 


\title{
PACIFIC JOURNAL OF MATHEMATICS
}

\author{
EDITORS
}

H. SAMELSON

Stanford University

Stanford, California 94305

J. DugunduI

Department of Mathematics

University of Southern California

Los Angeles, California 90007

RICHARD PIERCE

University of Washington

BASIL GORDON*

University of California

Seattle, Washington 98105

Los Angeles, California 90024

\section{ASSOCIATE EDITORS}

E. F. BeCKenbaCh

B. H. NeumanN

F. WOLF

K. YosHIDA

\section{SUPPORTING INSTITUTIONS}

\author{
UNIVERSITY OF BRITISH COLUMBIA \\ CALIFORNIA INSTITUTE OF TECHNOLOGY \\ UNIVERSITY OF CALIFORNIA \\ MONTANA STATE UNIVERSITY \\ UNIVERSITY OF NEVADA \\ NEW MEXICO STATE UNIVERSITY \\ OREGON STATE UNIVERSITY \\ UNIVERSITY OF OREGON \\ OSAKA UNIVERSITY \\ UNIVERSITY OF SOUTHERN CALIFORNIA
}

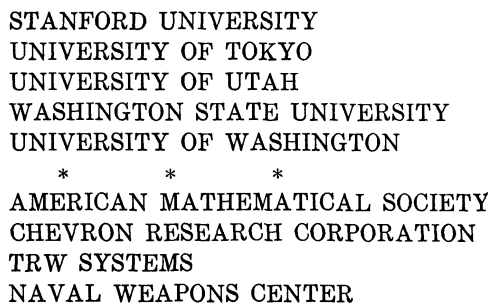

The Supporting Institutions listed above contribute to the cost of publication of this Journal, but they are not owners or publishers and have no responsibility for its content or policies.

Mathematical papers intended for publication in the Pacific Journal of Mathematics should be in typed form or offset-reproduced, double spaced with large margins. Underline Greek letters in red, German in green, and script in blue. The first paragraph or two must be capable of being used separately as a synopsis of the entire paper. It should not contain references to the bibliography. Manuscripts, in duplicate if possible, may be sent to any one of the four editors. Please classify according to the scheme of Math. Rev. 36, 1539-1546. All other communications to the editors should be addressed to the managing editor, Richard Arens, University of California, Los Angeles, California, 90024.

50 reprints are provided free for each article; additional copies may be obtained at cost in multiples of 50 .

The Pacific Journal of Mathematics is published monthly. Effective with Volume 16 the price per volume (3 numbers) is $\$ 8.00$; single issues, $\$ 3.00$. Special price for current issues to individual faculty members of supporting institutions and to individual members of the American Mathematical Society: $\$ 4.00$ per volume; single issues $\$ 1.50$. Back numbers are available.

Subscriptions, orders for back numbers, and changes of address should be sent to Pacific Journal of Mathematics, 103 Highland Boulevard, Berkeley, California, 94708.

PUBLISHED BY PACIFIC JOURNAL OF MATHEMATICS, A NON-PROFIT CORPORATION

Printed at Kokusai Bunken Insatsusha (International Academic Printing Co., Ltd.), 7-17, Fujimi 2-chome, Chiyoda-ku, Tokyo, Japan.

* Acting Managing Editor. 


\section{Pacific Journal of Mathematics}

\section{Vol. 32, No. 3 \\ March, 1970}

Shair Ahmad, Dynamical systems of characteristic $0^{+} \ldots \ldots \ldots \ldots \ldots$

Charles A. Akemann and Bernard Russo, Geometry of the unit sphere of a $C^{*}$-algebra and its dual............................ 575

Philip Bacon, The compactness of countably compact spaces ......... 587

Richard Blaine Barrar and Henry Loeb, On the continuity of the nonlinear Tschebyscheff operator ............................ 593

L. Carlitz, Factorization of a special polynomial over a finite field ....... 603

Joe Ebeling Cude, Compact integral domains .................... 615

Frank Rimi DeMeyer, On automorphisms of separable algebras. II . . . . . 621

James B. Derr, Generalized Sylow tower groups .................. 633

Raouf Doss, Some inclusions in multipliers ................... 643

Mary Rodriguez Embry, The numerical range of an operator........... 647

John Froese, Domain-perturbed problems for ordinary linear differential

operators..................................... 651

Zdeněk Frolík, Absolute Borel and Souslin sets ..................... 663

Ronald Owen Fulp, Tensor and torsion products of semigroups .......... 685

George Grätzer and J. Płonka, On the number of polynomials of an

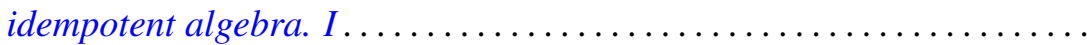

Newcomb Greenleaf and Walter Read, Positive holomorphic differentials on

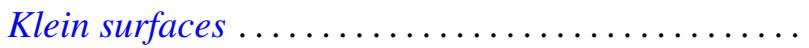

John Willard Heidel, Uniqueness, continuation, and nonoscillation for a second order nonlinear differential equation ................. 715

Leon A. Henkin, Extending Boolean operations................... 723

R. Hirshon, On hopfian groups .......................... 753

Melvin Hochster, Totally integrally closed rings and extremal spaces ..... 767

R. Mohanty and B. K. Ray, On the convergence of a trigonometric integral ..................................

Michael Rich, On a class of nodal algebras .................... 787

Emile B. Roth, Conjugate space representations of Banach spaces ........ 793

Rolf Schneider, On the projections of a convex polytope .............. 799

Bertram Manuel Schreiber, On the coset ring and strong Ditkin sets ...... 805

Edgar Lee Stout, Some remarks on varieties in polydiscs and bounded

holomorphic functions .........................

James Edward Ward, Two-groups and Jordan algebras . 